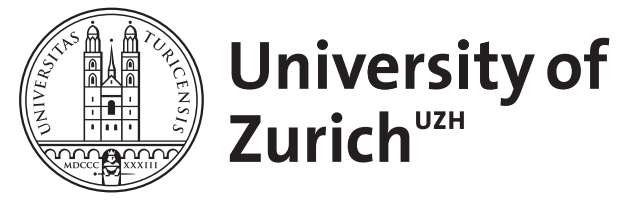

Zurich Open Repository and Archive

University of Zurich

University Library

Strickhofstrasse 39

CH-8057 Zurich

www.zora.uzh.ch

Year: 2018

\title{
Zwischen Abgrenzung und Aufklärung
}

Stuber, Selina

DOI: https://doi.org/10.24894/gesn-de.2018.75009

Posted at the Zurich Open Repository and Archive, University of Zurich

ZORA URL: https://doi.org/10.5167/uzh-184355

Journal Article

Published Version

Originally published at:

Stuber, Selina (2018). Zwischen Abgrenzung und Aufklärung. Gesnerus, 75(2):235-256.

DOI: https://doi.org/10.24894/gesn-de.2018.75009 


\section{Zwischen Abgrenzung und Aufklärung: Die Berichterstattung über die Antibabypille in der Schweizer Frauenzeitschrift Annabelle in den späten 1960er Jahren}

Selina Stuber

\section{Summary}

The birth control pill and its potential societal consequences were only discussed publicly in Switzerland from the mid-1960s onwards. While initial reports about the birth control pill were scarce, a change soon took place and reports became rather frequent. Women's magazines played a crucial role in that change. The Swiss women's magazine Annabelle not only provided its readers with knowledge concerning the contraceptive pill but also produced behavioral norms in terms of sexuality and society. In the essay at hand, the medical and sociopolitical context in which Annabelle thematized the new contraceptive pill in their issues from 1966 to 1968 will be examined.

Keywords: pill, contraception, sexuality, family planning, media analysis

\section{Zusammenfassung}

Die Antibabypille und ihre potentiellen gesellschaftlichen Auswirkungen wurden in der Schweiz erst ab Mitte der 1960er Jahre öffentlich diskutiert. Die Berichterstattung über die Antibabypille wandelte sich von einer anfänglichen Nicht-Thematisierung zu einer regen Berichterstattung. In diesem $\mathrm{Zu}-$ sammenhang kam Frauenzeitschriften eine zentrale Rolle zu. Die Schweizer Frauenzeitschrift Annabelle vermittelte ihren Leserinnen nicht nur Wissen über das neue Verhütungsmittel, sondern formulierte auch gesellschaftliche und sexuelle Verhaltensnormen. Im vorliegenden Aufsatz wird untersucht in

Selina Stuber, Stauffacherstrasse 98, 8004 Zürich, selina.s@gmx.net 
welchem medizinischen und gesellschaftspolitischen Rahmen die Annabelle das neue Verhütungsmittel in den Jahren 1966 bis 1968 thematisierte.

Schlagworte: Antibabypille, Pille, Verhütung, Sexualität, Familienplanung, Frauenzeitschrift, Medienanalyse

«Jedermann weiss, was die 'Pille' ist. Sie ist winzig klein im Vergleich mit der Atombombe, aber ihre Wirkung auf unsere Gesellschaft kann noch verheerender sein.» ${ }^{1}$

Das Zitat stammt aus der Märzausgabe der Schweizer Frauenzeitschrift Annabelle von 1968 und ist einem Artikel der Amerikanerin Pearl S. Buck entnommen, in dem sie sich mit den Auswirkungen der Antibabypille ${ }^{2}$ auf Individuum und Gesellschaft auseinandersetzt. Durch den Vergleich mit der Atombombe zeichnete die Autorin ein Bild der Wirkungsmacht der Pille, das bedrohlicher nicht sein könnte. Das Aufkommen des hormonellen Kontrazeptivums in den frühen 1960er Jahren sorgte in weiten Kreisen für alarmierende Einschätzungen, da die bestehende Sexualmoral und somit auch die gesellschaftliche Ordnung in Gefahr zu sein schienen. In einem Artikel der Neuen Zürcher Zeitung (NZZ) vom 15. September 1966 wurde das «Gefahrenpotential» der Pille ebenfalls im gesellschaftlichen Bereich verortet und wie folgt eingeschätzt: «Die wesentliche Problematik dieser hochwirksamen Präparate zur Empfängnisverhütung liegt unseres Erachtens nicht auf pharmakologischem, sondern auf ethischem, sozialem und demographischem Gebiet.» ${ }^{3}$ Das neue Verhütungsmittel wurde zur Zeit seiner medialen Einführung Mitte der 1960er Jahre mit verschiedensten Themen und Interessen - wie Familienplanung, der Befreiung der weiblichen Sexualität sowie Veränderungen der Sexualmoral - in Verbindung gebracht. ${ }^{4}$

Neben diesen polarisierenden Annahmen zur hormonellen Verhütung die zwar den öffentlichen Diskurs prägten, sich jedoch nicht immer bewahrheiteten - hatte die Antibabypille auch ganz konkrete Auswirkungen auf weibliche Körper und Sexualität. ${ }^{5}$ Die Einnahme der Antibabypille ermög-

1 Das Zitat stammt aus der Annabelle Nr. 448, März 1968, S. 8. Künftig wird auf die Annabelle direkt im Fliesstext in Klammern mit Nummer, Monat/Jahr sowie Angabe der Seitenzahl verwiesen.

2 Im vorliegenden Beitrag wird häufig die Kurzform «Pille» zur Bezeichnung der Antibabypille verwendet.

3 Schreiner, W.E.: Die «Pille» zur Empfängnisverhütung, in: NZZ, Nr. 3880, Morgenausgabe, Blatt 5, 15.09.1966.

4 Suter 1996, 3.

5 Die Forschung ist sich einig, dass der Einfluss der Pille auf Veränderungen wie das weibliche Sexualverhalten oder die «sexuelle Revolution» Ende der 1960er Jahre überschätzt wurde und teilweise noch immer wird (vgl. dazu Watkins 1998, 2; Marks 2001, 3; Niethammer/Satjukow 2016, 15 sowie Wajcman 1994, 103). 
lichte Frauen erstmals eine sichere Kontrolle über die eigene Fertilität und somit eine grössere Selbstbestimmung. ${ }^{6}$ Die Pille konnte als präventiver Akt bei der Familienplanung eingesetzt werden und hatte somit einen Einfluss auf die Bildung «präventiver Selbste». ${ }^{7}$ Durch die Pille veränderten sich für viele Frauen auch der Umgang und die Wahrnehmung von Pharmakologie und Arzneimittelanwendung. ${ }^{8}$ Die Pille wurde nicht nur als Medikament im herkömmlichen Sinn zur Regulierung des weiblichen Zyklus, die pathologische Erscheinungsformen wie etwa Zyklusschwankungen ausgleichen sollte, zugelassen. Im Fall der Anwendung als hormonelles Kontrazeptivum lag der Verabreichung der Pille kein diagnostiziertes Krankheitsbild zugrunde. Gesunden Frauen wurde so durch die tägliche Einnahme chemischer Wirkstoffe - der Hormone Gestagen und Östrogen - ermöglicht, medikamentös in die Familienplanung einzuwirken. ${ }^{9}$ Die Tatsache, dass die Einnahme der Pille in der Schweiz rezeptpflichtig war, bedeutete für Frauen zudem, regelmässige Arztbesuche wahrzunehmen, auch wenn sie sonst gesund waren. ${ }^{10}$ Bei der Entscheidung einer geeigneten Verhütung waren Frauen - trotz der selbstbestimmten Anwendung - abhängig von der Einschätzung einer ärztlichen Kompetenz. Ärzte waren ab den 1950er Jahren die bestimmenden Experten im Bereich der Fertilität und übten daher in wesentlichen Belangen Kontrolle über weibliche Körper aus. ${ }^{11}$

Auch wenn das neue Verhütungsmittel erstmals eine längerfristige Loslösung der Sexualität von der Fortpflanzung ermöglichte, ${ }^{12}$ wurden diese zwei Themenbereiche in den Medien sehr eng geführt. So löste die Verfügbarkeit der Antibabypille «heftige öffentliche Debatten» über die bestehende Sexualmoral und weibliche Sexualität aus, wie Staupe und Vieth für Deutschland festhalten. ${ }^{13}$ In der Schweiz wurden diese Debatten erst ab Mitte der 1960er Jahre öffentlich verhandelt - obwohl die ersten hormonalen Kontrazeptiva schon seit 1962 auf dem Markt waren. ${ }^{14}$ Dieser Umstand hängt damit zusammen, dass sich die Akzeptanz, Empfängnisverhütung und ihre möglichen Auswirkungen in einer medialen Öffentlichkeit zu thematisieren, nur langsam entwickelt hat und erst ab Mitte der 1960er Jahre gesellschaftlich toleriert wurde. ${ }^{15}$ Bemerkenswert ist zudem, dass eine Dis-

6 vgl. Marks, 3.

7 Lengwiler/Madarasz 2010, 16.

8 Vgl. Suter 1996, 30.

9 Vgl. Duden 1996, 74.

10 Vgl. Suter 1996, 30.

11 Vgl. Watkins 1998, 3.

12 Joris/Witzig 20014, 297.

13 Staupe/Vieth 1996, 11.

14 Vgl. Tanner 2015, 361.

15 Vgl. Joris/Witzig 20014, 297. 
kussion über Verhütung im Allgemeinen erst durch die Pille aufgekommen ist. Zuvor wurden solche Themen nur im medizinischen Kontext oder in einem privateren Rahmen besprochen. ${ }^{16}$ Die Historikerin Monica Suter führt in ihrer Dissertation zum medizinischen Diskurs über Empfängnisverhütung in der Schweiz aus, dass die Pille ab Mitte der 1960er Jahre vermehrt im Zusammenhang von Sexualität thematisiert wurde. ${ }^{17}$ Suter schreibt weiter, dass in der Schweizer Frauenzeitschrift Annabelle ab 1967 «plötzlich intensiv» über Sexualität gesprochen wurde. ${ }^{18}$ Sie führt ihre Beobachtung jedoch nicht weiter aus und nennt keine Gründe für diesen «plötzlichen» Wandel.

An diesem Punkt setzt der vorliegende Beitrag ein und geht Suters Beobachtung einer veränderten Berichterstattung in der Zeitschrift Annabelle nach. Dafür werden die Ausgaben von 1966 bis 1968 in Hinblick auf die Berichterstattung über die Pille eingehend analysiert und aufgezeigt, wie die Pille in der Öffentlichkeit thematisiert wurde. Die Eingrenzung auf diesen Untersuchungszeitraum wurde nach einer systematischen Durchsicht der Annabelle von 1961 bis 1970 festgelegt. Dabei kann festgestellt werden, dass Verhütung bis 1966 in der Frauenzeitschrift nicht thematisiert und das Thema Sexualität nur vereinzelt besprochen wurde.

Neben der ersten zagen Berichterstattung wird die damit im Zusammenhang stehende aufkommende Medialisierung der Sexualität in der Frauenzeitschrift textnah nachgezeichnet. Besonderes Augenmerk wird auf die zwei Themenbereiche Empfängnisverhütung und Sexualität sowie auf die Diskussion, wie sich diese beiden Themen im medialen Kontext gegenseitig bedingen, gelegt. In der Untersuchungsperiode vollzog sich in der $A n$ nabelle ein dynamischer Wandel von einer anfänglichen Nicht-Thematisierung von Verhütung und Sexualität bis hin zu einer regen Berichterstattung. Wie im Folgenden dargelegt wird, veränderten sich in diesen drei Jahren nicht nur die Art und Häufigkeit der Berichterstattung, sondern auch die Akteure, die in der Annabelle zu Wort kamen. Der Wandel der SprecherInnenrollen vollzog sich von der Berichterstattung von Psychotherapeuten, über Mediziner bis hin zur Annabelle-Redaktion, die in beratender Funktion fungierte.

Pearl S. Bucks Aussage im einleitenden Zitat «Jedermann weiss, was die 'Pille' ist.» verweist auf einen weiteren Aspekt im Zusammenhang mit der Berichterstattung über die Pille. So impliziert ihre Feststellung, dass 1968 davon ausgegangen werden konnte, dass die meisten Menschen über

16 Vgl. ebd., 297.

17 Suter 1996, 2.

18 Ebd., 146. 
die Existenz einer Antibabypille und deren Funktion unterrichtet waren. Demnach hat das Wissen über die Pille innerhalb weniger Jahre die Fachkreise verlassen und war im Bewusstsein der Bevölkerung angekommen. Bei der Bekanntmachung der Pille in der breiten Öffentlichkeit spielten Zeitschriften wie die Annabelle eine zentrale Rolle, da die Pille als rezeptpflichtiges Medikament unter einem Werbeverbot stand. Informative Texte über das Verhütungsmittel waren jedoch erlaubt und so wurde vor allem in Zeitschriften - oft in einem aufklärerischen Duktus - über die Antibabypille berichtet. ${ }^{19}$ In der Annabelle wurde die Antibabypille von ihrer Einführung an als Mittel zur Kontrazeption vorgestellt und nicht etwa zur Linderung von Menstruationsbeschwerden oder Hautproblemen - wie die Pille beispielsweise in der BRD zu Beginn der 1960er Jahre diskutiert wurde. ${ }^{20}$

Unabhängig von Suters Bezugnahme bietet sich die Annabelle als Quellengrundlage an. So gehörte die Annabelle Mitte der 1960er Jahre zu den auflagenstärksten Schweizer Frauenzeitschriften und hatte als Leitmedium für Frauenthemen grossen Einfluss auf die Meinungsbildung ihrer Leserinnen. ${ }^{21}$ Es kann zudem davon ausgegangen werden, dass sich viele Frauen aus bürgerlichen Kreisen in der Schweiz erst durch die Berichterstattung in der Annabelle detailliert über die neue, hormonelle Verhütungsmethode informierten. ${ }^{22}$ Von der Existenz der Antibabypille hatten die Leserinnen wahrscheinlich jedoch schon über andere Medien gehört, so hat beispielsweise die Neue Zürcher Zeitung ab 1962 vereinzelt ${ }^{23}$ über die Pille berichtet. Die Annabelle brachte sich also nicht im Rahmen der Erstberichterstattung ${ }^{24}$ in den Diskurs ein, sondern vermittelte (Fach-)Wissen in Form von ausführlichen Reportagen und Artikeln.

19 Vgl. Suter 1996, 122.

20 Vgl. Silies 2016, 195.

21 Vgl. Hediger 2004, 59; Christen/Gisler/Heller 1992, 9.

22 Das Zielpublikum der Annabelle war vor allem gut situierte Frauen aus dem städtischen Umfeld (vgl. Christen/Gisler/Heller 1992, 8).

23 Vgl. dazu folgende Artikel aus der Neuen Zürcher Zeitung (NZZ): E.M.: Englische Diskussion um gefährliche Medikamente, in: NZZ, Nr. 3021, Abendausgabe, Blatt 2, 06.08.1962; E.M.: Morallehre und Familienplanung. Diskussion im britischen Katholizismus, in: NZZ, Nr. 2022, Mittagsausgabe, Blatt 2, 11.05.1964; Schreiner, W.E.: Die «Pille» zur Empfängnisverhütung, in: NZZ, Nr. 3880, Morgenausgabe, Blatt 5, 15.09.1966; o.A.: Illegaler Pillenversand in Deutschland, in: NZZ, Nr. 5575, Morgenausgabe, Blatt 5, 24.12.1966.

24 Weiter gab es die Möglichkeit, sich über verschiedene (medizinische) Ratgeber zur Geburtenkontrolle in der Ehe über das hormonale Kontrazeptivum zu informieren (vgl. Suter 1996, 3). 


\section{A nnabelle: Zwischen bürgerlicher und moderner Ausrichtung}

1938 wurde mit der Annabelle die erste klassische Frauenzeitschrift der Schweiz gegründet. ${ }^{25} \mathrm{Zu}$ dieser Zeit war die Annabelle das einzige Medium in der Schweiz, das sich inhaltlich auf Frauenthemen fokussierte und weibliche Anliegen öffentlich machte. Themen, die bis dahin der privaten Sphäre zugeschrieben worden waren - der Bereich der Hausfrauen und Mütter -, wurden so Teil der massenmedialen Öffentlichkeit. ${ }^{26}$ Der kommerzielle Erfolg der Annabelle setzte erst ab 1943 richtig ein, danach blieb sie jedoch über lange Zeit die auflagenstärkste Frauenzeitschrift der Schweiz. ${ }^{27}$ In den 1950er und 1960er Jahren nahm die Annabelle im Kreis ihrer Leserinnen eine wichtige Rolle ein, da sie Vorstellungen davon vermittelte, was Weiblichkeit und «Frau-Sein» in der Nachkriegszeit bedeuteten und den Frauen so eine Orientierungshilfe bot. ${ }^{28}$ Themen wie Disziplin, Aussehen und Liebenswürdigkeit wurden als Grundlage für ein erfolgsversprechendes «Frau-Sein» etabliert. ${ }^{29}$ Hinter diesen Vorstellungen von Weiblichkeit stand ein «zutiefst bürgerlicher Habitus», der sich erst in den 1960er Jahren allmählich lockerte und moderneren Ansichten wich. ${ }^{30}$ Annabelle vermittelte ein Frauenbild, das stark an der Familie orientiert war und daher die Frau als Mutter, Haus- und Ehefrau ansprach. ${ }^{31}$

In der zweiten Hälfte der 1960er Jahre - die Zeitspanne, die im vorliegenden Beitrag analysiert wird - war die Annabelle hin- und hergerissen zwischen konservativer und moderner Ausrichtung: Einerseits sollten die klassischen Inhalte und alten Strukturen der Zeitschrift bestehen bleiben, andererseits musste sie sich der gesellschaftlichen Realität anpassen und gesellschaftspolitische Themen aufgreifen. So wurden neben einem Frauenbild, das sich stark an der Familie orientierte, zunehmend «neue» Themen wie Teilzeitarbeit, Scheidung, Gleichberechtigung und das Frauenstimmrecht aufgegriffen. ${ }^{32}$ Dieser inhaltliche Kurswechsel beruht zu einem grossen Teil auf einer wirtschaftlichen Notwendigkeit: Ab Mitte der 1960er

25 Christen definiert die klassische Frauenzeitschrift als Erscheinung der 1930er Jahre, als die Frau als Konsumentin entdeckt wurde. Inhaltlich soll über «alle Interessen der Frauenwelt» berichtet werden. Der Fokus lag vorerst auf dem Themengebiet der Mode. (vgl. Christen/ Gisler/Heller 1992, 10)

26 Vgl. ebd., 11.

27 Vgl. Christen/Gisler/Heller 1992, 8. Laut Jakob Tanner (vgl. Tanner 2015, 369) stieg die Auflage der Annabelle zu Beginn der 1960er Jahre von 10'000 zu 100'000 Exemplaren und erreichte 1968 mit 115'000 Exemplaren einen Spitzenwert.

28 Vgl. dazu Hediger 2004, 59; Christen/Gisler/Heller 1992, 9.

29 Vgl. ebd., 9.

30 Vgl. ebd.

31 Vgl. Christen/Gisler/Heller 1992, 11.

$32 \mathrm{Zu}$ diesem Abschnitt vgl. ebd., 13. 
Jahre stieg der Anteil von Frauenzeitschriften auf dem Schweizer Markt an und die Annabelle musste auf die stärkere Konkurrenzsituation reagieren. ${ }^{33}$ Gegen Ende des Jahrzehnts verstärkte sich der Konkurrenzdruck und die Annabelle verlor vor allem jüngere Leserinnen an ausländische Zeitschriften wie dem französischen Frauenmagazin Elle. ${ }^{34}$

In der Untersuchungsperiode von 1966 bis 1968 erschien die Annabelle zweimal monatlich und der Seitenumfang variierte zwischen 100 bis 300 Seiten. ${ }^{35}$ Die Rubriken blieben in diesen Jahren relativ konstant und es gab nur kleinere Änderungen. Für die Analyse waren vor allem das Editorial, verschiedene Reportage- oder Artikelserien sowie die Rubrik «Welt der Frau» relevant. Prägendste Figur der Belegschaft war Eva Maria Borer, die ab 1960 die Chefredaktion in der Annabelle übernahm. Borer (1905-1987) war eine deutsch-schweizerische Journalistin und verfasste verschiedene Bücher, darunter eine Autobiographie, Kochbücher sowie kulturhistorische Werke. 1933 wurde sie als politische Gefangene in Deutschland inhaftiert und emigrierte nach ihrer Entlassung 1936 in die Schweiz. Nach dem Zweiten Weltkrieg war sie für verschiedene Frauenzeitschriften tätig und schrieb über Mode, Kochen und Kultur. In der Annabelle trug sie als Chefredaktorin zur Professionalisierung der Zeitschrift bei, indem sie strukturelle sowie personelle Veränderungen einleitete. Sie trat als Lebensberaterin auf, beantwortete Leserinnenfragen und äusserte in der Rubrik «Meine Meinung» ihre persönliche Einschätzung zu aktuellen Themen, so auch in Bezug auf die Berichterstattung über die Pille. Durch ihre Präsenz verlieh sie der Annabelle eine Stimme und ein Gesicht. ${ }^{36} 1966$ wurde die Entscheidung getroffen, Hans Gmür als zweiten Chefredaktor anzustellen, um vermehrt auch ein männliches Publikum anzusprechen. ${ }^{37}$ Eine weitere Rubrik, die auch in der Analyse mehrmals beigezogen wird, ist Dr. Fritz Tanners Kolumne «Aus der Praxis des Beraters». Darin berichtete der Psychotherapeut aus seiner Eheberatungs- und Psychotherapiepraxis und ging auf aktuelle gesellschaftliche «Probleme» ein. ${ }^{38}$

33 Vgl. Hediger 2004, 59.

34 Vgl. Christen/Gisler/Heller 1992, 12.

35 Bis 1968 wurde die Zeitschrift von der Annabelle-Verlagsgesellschaft herausgegeben, danach ging sie an den Jean Frey Verlag über (vgl. ebd., 68).

36 Vgl. ebd., 60f.

37 Vgl. ebd.

38 Vgl. Peter-Kubli, Fritz Tanner, in HLS online, http://www.hls-dhs-dss.ch/textes/d/D6704. php [Stand 31.08.2018]. 


\section{Blick nach Amerika: Thematisierung «fremder» Sexualität}

Bis 1966 wurden Verhütung und Sexualität in der Annabelle praktisch gar nicht thematisiert. Lediglich unter dem Aspekt der Erotisierung der Ehe wurden zu Beginn der 1960er Jahre sexuelle Themen am Rande besprochen. Dabei ging es darum, den Leserinnen beizubringen - diese pädagogische Haltung gegenüber der Leserschaft war typisch für die Annabelle der Nachkriegszeit -, was Sex-Appeal und weibliche Sexualität bedeuteten. Die Anstrengungen waren gänzlich auf männliche Vorstellungen ausgerichtet und sollten dabei helfen, die Ehe aufzuwerten und zu stabilisieren. ${ }^{39}$ Im Juli 1966 änderte sich diese Tatsache mit einer vierteiligen Reportage: «Liebe und SexKult in Amerika. Symptome und Maskottchen der Sexualrevolution» (Nr. 404, Juli 1966) war der erste Beitrag in der Annabelle, der sich ausführlich und explizit mit dem Thema der Sexualität befasste.

Im Bericht geht der Verfasser, der amerikanische Psychotherapeut Dr. Paul J. Stern, davon aus, dass eine «sogenannte Sexualrevolution» (ebd., 37) die USA erfasst habe und beschreibt anhand verschiedener Erfahrungsbeispiele, wie diese beschaffen sei. Dabei wird die «neue», öffentliche Sexualität aus einer sittlich-moralischen Perspektive kritisiert und eine Definition von richtiger und falscher Sexualität formuliert. Nach Stern würde die Sexualität durch ihre Verbindung mit den Massenmedien kommerziell ausgeschlachtet und ihres «dynamischen Gehaltes» (ebd.) beraubt. Der Abbau von Sexualtabus führe zudem zu sexueller Frühreife, die befreitere Sexualität zu einer «Zersplitterung der Persönlichkeit» (ebd., 38). Stern ist zurückhaltend mit der Nennung von Gründen für diese Entwicklung und so wird auch die neue hormonelle Verhütungsmethode nicht erwähnt. Amerikanische Sexualität sei dadurch gekennzeichnet, dass sie in erster Linie fun sein solle, die «echte» Sexualität - die verbunden sei mit intensiven Gefühlen und emotionaler Nähe zum Partner - spiele im «Sex-Kult» keine Rolle mehr und werde so entwertet. Stern deutet die «Befreiung der Sexualität» negativ, da die Trennung der Sexualität vom Reproduktionsprozess in seinen Augen auch zu einer Trennung von Sexualität und Liebe führe. Seinen Ausführungen liegt die Idealvorstellung zugrunde, dass Sexualität, Liebe und Fortpflanzung eine Einheit bilden. Abweichungen von dieser «Trinität» werden von ihm abgelehnt und abwertend psychologisiert.

Das Annabelle-Team kam bei dieser Reportage selbst nicht zu Wort nicht einmal mit einem einleitenden Kommentar -, sondern überliess die Einschätzung gänzlich einem «berühmten USA-Gelehrten»(Nr. 404,

39 Vgl. Gisler/Christen 1994, 193. 
Juli 66, 37). Indem der Leserschaft vermittelt wurde, dass es sich bei diesem Wissen über Sexualität um «Experten-Wissen» eines Psychotherapeuten handelte, werden die Artikel legitimiert und die Annabelle kann sich so von potentiell problematischen Inhalten distanzieren. Diese Art der Berichterstattung verweist zudem auf eine Verwissenschaftlichung auf dem Gebiet der Sexualität, wobei sich vor allem die Medizin sowie Psychologie als wortführende Instanzen behaupten konnten. ${ }^{40}$ Erkenntnisse aus den Bereichen der Psychologie und Sexologie prägten in den 1960er Jahren neue sexuelle Verhaltensregeln, die über Massenmedien und Aufklärungsliteratur verbreitet wurden und so einen grossen Teil der Gesellschaft erreichten. ${ }^{41}$ Dieses stark (natur-)wissenschaftlich geprägte Sprechen über Sexualität steht in Zusammenhang mit der Publikation der beiden Kinsey-Reporte ${ }^{42}$ von 1948 und 1953, die auch in Europa grosse Resonanz auslösten. ${ }^{43}$ Darin beschrieb Kinsey Sexualität erstmals in einer grossen Variationsbreite und in verschiedenen Ausprägungen und versuchte so Vorurteile gegenüber «unnatürlich» angesehenen Praktiken abzubauen. Die Reporte führten zu einer Öffnung des Normalitätsspektrums und hatten Einfluss darauf, wie in der Öffentlichkeit über sexuelle Themen auch in der Schweiz gesprochen wurde. ${ }^{44}$

Die Annabelle fügte sich mit der Reportage in diese Entwicklung ein und liess einen Psychotherapeuten zu Wort kommen. Dabei ist die Tatsache bedeutend, dass in der Reportage amerikanische Entwicklungen, also der Umgang mit Sexualität in einer anderen Kultur, aufgezeigt werden. $\mathrm{Zu}$ diesem Zeitpunkt wurde in der Annabelle also nicht die «eigene» Sexualität thematisiert, sondern es fand lediglich eine Annäherung an die Thematik in einem Duktus der Abgrenzung statt. Aber gerade die Abgrenzung zur eigenen Kultur durch das Aufgreifen von Trends aus den USA gab der AnnabelleRedaktion Anlass, Sexualität erstmals ausführlicher und öffentlich zu thematisieren ohne die hiesige Sexualmoral direkt anzugreifen. Steinbacher zufolge war der Fokus auf Amerika im öffentlichen Sprechen über Sexualität zentral, «als sich die Umbrüche, Neuerungen und Bedrohungen, die von Sexualität ausgingen, massgeblich auf die 'Kultur von drüben' bezogen».45

Diese Annäherung kann als erster Schritt in Richtung einer offeneren Thematisierung von Sexualität gedeutet werden. Möglicherweise bewirkte

40 Vgl. Joris/Witzig 20014, 291f.

41 Vgl. Eder 2010, 105.

42 Als Kinsey-Reporte werden zwei Publikationen des amerikanischen Sexualwissenschaftlers Alfred Charles Kinsey bezeichnet, in denen statistische Untersuchungen zu den körperlichen Vorgängen der Sexualität gesammelt wurden. Der 1948 publizierte Report hatte die männliche, der von 1953 die weibliche Sexualität zum Thema.

43 Siehe dazu bspw. Steinbacher, 2011.

44 Vgl. Joris/Witzig 20014, 294 sowie Bänziger/Beljan/Eder et al. 2015, 13.

45 Steinbacher 2011, 11. 
die pessimistische Einschätzung zum Zerfall der Sexualnormen in den USA zudem, dass die Entwicklungen in der Schweiz weniger dramatisch eingeschätzt wurden. In der Annabelle wurde wiederholt über gesellschaftliche Entwicklungen aus den USA berichtet. Diese Ausrichtung erklärte Fritz Tanner im Mai 1968 im Rahmen seiner Beratungskolumne und führte aus, dass der Blick nach Amerika eine Trendeinschätzung ermögliche, da «typisch amerikanische» Verhaltensweisen oft nur kurze Zeit später auch in der Schweiz beobachtet werden könnten (vgl. Nr. 452, Mai 68, 10).

\section{Aufklärung und Information}

Anschliessend an die Amerika-Reportage folgte ab Oktober 1966 eine weitere vierteilige Serie, nun unter dem Titel «Hormone lenken das Frauenleben». Darin wurde die Leserschaft der Annabelle zum ersten Mal detailliert und ausführlich über die neue Möglichkeit der Geburtenkontrolle informiert. Die Annabelle setzte mit ihrer Berichterstattung im Vergleich mit anderen deutschsprachigen Zeitschriften erst relativ spät ein, so wurde beispielsweise in der westdeutschen Frauenzeitschrift Constanze schon ab 1964 ausführlich über die Pille berichtet. ${ }^{46}$ Auch die Artikel in der Reihe «Hormone lenken das Frauenleben» wurden nicht vom Redaktionsteam der Annabelle selbst verfasst, sondern das Sprechen wurde wiederum Experten überlassen, in diesem Fall dem österreichischen Medizinjournalisten Dr. Eric Weiser. Im Lead des ersten Teils «Von Monat zu Monat» wurden Hormone als «mächtige Naturkräfte» (Nr. 412, Okt 66, 158) beschrieben, welche die Fortpflanzung und das Leben der Menschen bestimmen würden. In wissenschaftlichem Ton, der mit vielen medizinischen Fachausdrücken gespickt war, wurden die Leserinnen über den weiblichen Hormonhaushalt und die möglichen Einflüsse auf die Psyche informiert.

Diese im sachlich-informativen Duktus gehaltene Berichterstattung in der Annabelle kann in einen grösseren Kontext eingeordnet werden. Ab den späten 1950er Jahren erschienen in medizinischen Handbüchern und Fachzeitschriften Artikel über die Wirkungsweisen der hormonellen Kontrazeptiva. Dabei wurde die Relevanz der Pille für Gesellschaft und Individuum hervorgehoben und die zentrale Rolle der Ärzteschaft in diesem Zusammenhang betont. ${ }^{47}$ Als die hormonelle Verhütungsmethode auch in der breiteren Medienöffentlichkeit diskutiert wurde, traten Ärzte mit ihrem Fach-

46 Silies 2016, 198.

47 zu diesem Abschnitt vgl. Suter 1996, 3. 
wissen auf, indem sie sich als Experten in Interviews und Artikeln zu Wort meldeten. Die Präsenz des medizinischen Diskurses - verbunden mit einer Stärkung der Rolle der Ärzteschaft im Bereich der Verhütung - war auch in der Annabelle gegenwärtig.

Zum Schluss des ersten Artikels «Von Monat zu Monat» erwähnte Weiser die Möglichkeit der Geburtenkontrolle. Er führte jedoch nicht weiter aus, inwiefern diese mithilfe von Hormonen gesteuert werden konnte. $\mathrm{Zu}$ dem wurde in diesem Zusammenhang der Begriff der Familienplanung eingeführt und betont, dass dieser für Frauen und Männer unterschiedliche Bedeutungen trüge: «Für den Mann ist die Familienplanung ein menschliches, soziales und wirtschaftliches Problem: für die Frau aber geht es hier um weit mehr - um ihr Recht auf den eigenen Körper» (ebd., 159). Familienplanung wird an dieser Stelle als komplexer Interessenbereich vorgestellt, der geschlechterspezifische Konnotationen erfährt. Die Vorstellung des Konzepts der Familienplanung ging einer Thematisierung von Verhütungsmitteln voraus. Die Besprechung der Pille war somit fest im Narrativ der Familienplanung verankert.

Die Annabelle liess zwar den ersten Teil von der Hormon-Serie unkommentiert, in der Rubrik «Meine Meinung» äusserte sich Eva Maria Borer jedoch im Rahmen einer Besprechung des Buchs Die vollkommene Frau, verfasst vom amerikanischen Frauenarzt Dr. Robert A. Wilson, zum «Oestrogen-Rummel» (vgl. ebd., 35) und stellte eine Verbindung von Hormonen und Empfängnisverhütung her. Borer fasste zusammen, dass Dr. Wilson die Forderung formuliere, dass Ärzte die Pille allen Frauen verschreiben sollten, die sie anwenden möchten. Anschliessend teilte Borer der Leserschaft ihre persönliche Meinung zu dieser Handhabung mit. Sie äusserte sich kritisch zu den neuen Behandlungsmethoden und betonte, dass eine Hormonbehandlung nur in Absprache mit einem Arzt entschieden werden solle, alles andere sei verantwortungslos. Borer erwähnte zudem ausdrücklich, dass sie ihre Meinung in Rücksprache mit Ärzten gebildet hätte. Es ist daher nicht weiter erstaunlich, dass sie in ihrer Stellungnahme die Position der Ärzte als Experten für Geburtenkontrolle und Familienplanung hervorhebt und so - ob bewusst oder unbewusst sei dahingestellt - die ärztliche Kompetenz in diesem Bereich stärkt.

«Geburtenregelung zwischen gestern und morgen» lautete der Titel des zweiten Teils der Hormon-Serie. Darin wurden, wie schon der Titel verrät, bewährte und neue Verhütungsmittel mit ihren Vor- und Nachteilen vorgestellt. Im einführenden Lead akzentuierte die Redaktion der Annabelle die Bedeutung, die dem hormonellen Verhütungsmittel zukam und beschrieb die Vorteile der gegenwärtigen Situation für die «emanzipierte Frau» wie folgt: 
[...] endlich [besteht] die Möglichkeit, über ihren Körper und ihr Schicksal nach freiem Willen zu verfügen und sich unerwünschter Mutterschaft zu entziehen, ohne ihre Gesundheit ernstlich aufs Spiel zu setzen oder auf ein normales Liebes- und Eheleben verzichten zu müssen. (Nr. 413, Nov 66, 128)

Die Pille wird in dieser Einführung im Kontext von Befreiung und weiblicher Selbstbestimmung beschrieben, die ein «normales Liebes- und Eheleben» ermögliche (ebd.). Auch wenn nicht weiter ausgeführt wird, wie diese Normalität konkret aussieht, so impliziert die Aussage, dass die Pille als Voraussetzung für ein funktionierendes Eheleben fungiert. Die damit suggerierte sexuelle Selbstbestimmung wird hier auf die Familienplanung innerhalb der Ehe enggeführt. Im Unterschied zum restlichen Beitrag wird hier das neue Verhütungsmittel vom Redaktionsteam als Mittler zwischen freier, selbstbestimmter weiblicher Sexualität und einem traditionellen Familienbild in Einklang gebracht. Dieser Lead unterscheidet sich sprachlich und inhaltlich stark vom restlichen Beitrag, der vom Medizinjournalisten Dr. Eric Weiser verfasst wurde und von einer medizinisch-wissenschaftlichen Sprache geprägt ist.

Im folgenden Überblick über die Verhütungsmethoden werden ältere Mittel gegenüber den hormonellen Kontrazeptiva abgewertet, da sie keine hundertprozentige Sicherheit bieten würden. Die Pille wird als erste Stufe einer zukünftigen Entwicklung von hormonellen Verhütungsmitteln ${ }^{48}$ umschrieben. Mit den verheissungsvollen Worten «[...] die Wissenschaft steht nicht still.» (ebd., 129) wird die Pille in einen medizinischen Fortschrittsdiskurs eingeordnet. Die Pille wurde als Verhütungsmittel mit «doppelter Sicherheit» (ebd., 128) angepriesen, da sie eine empfängnisverhütende Wirkung habe, zugleich aber auch die vollständige Fruchtbarkeit nach Abbruch der Behandlung garantiere. Die abschliessende Aussage «Die Möglichkeiten zur Familienplanung sind also heute bereits zahlreich und mannigfaltig» (ebd.) macht deutlich, dass die Pille sowie andere Verhütungsmittel auch im medizinischen Expertenmodus klar dem Kontext der ehelichen Familienplanung zugeordnet wurden. Diese enge Verschränkung von Medizin, Familienplanung und Verhütung verweist ebenfalls darauf, dass der medizinische Diskurs ${ }^{49}$ um Verhütung Teil des öffentlichen Diskurses wurde. Da der Artikel von einem Medizinjournalisten verfasst wurde, ist diese Verbindung naheliegend. Weiter wurde in dieser Ausgabe der Begriff der «Anti-BabyPille» eingeführt, vorher wurde ausschliesslich von hormonellen Verhütungsmitteln gesprochen. Das Verorten der Antibabypille im Kontext von

48 Es wird ausgeführt, dass an «Nidationshemmern», einer Monatspille sowie befruchtungsverhütenden Pillen für Männer geforscht wird (Nr. 413, Nov 66, 129).

49 Vgl. Suter 1996, 122. 
Familienplanung ermöglichte es der Annabelle, über Verhütung zu sprechen, ohne dabei (weibliche) Sexualität direkt zu thematisieren.

Nach dieser ersten Aufklärungsserie über die Pille wurde das Thema der Empfängnisverhütung bis im Mai 1967 nicht mehr behandelt. ${ }^{50} \mathrm{Im}$ Interview «Der Vater der Pille» - von einem französischen Journalisten verfasst - kam dann jedoch der Experte schlechthin zu Wort: der amerikanische Wissenschaftler Gregory Pincus. Er wurde als «Mister Pill», als «Schöpfer der Pille», als «eine der grössten Kapazitäten der Welt auf den Gebieten der Fortpflanzung und der weiblichen Sterilität» (Nr. 426, Mai 67, 100) umschrieben. Im Interview stand dann jedoch der Forscher als Mensch im Mittelpunkt. Pincus sprach über seinen Werdegang, seine familiäre Situation und gab persönliche Einschätzungen rund um das Thema der hormonellen Verhütung ab. In einer Frage wurde das Potenzial der Pille angesprochen: «Und diese Pille kann das Verhalten und die Rolle der Frau in der Gesellschaft völlig verändern. Sie kann zur sexuellen Freiheit der Frau führen. Sind Sie für eine solche Freiheit?» (ebd., 103). Pincus beantwortete die Frage mit einem klaren Nein. Er merkte jedoch an, dass er ebenso wenig die sexuelle Freiheit des Mannes als erstrebenswert erachte. Für ihn werde der Einfluss der Sexualität auf Individuum, Familie und Gesellschaft gemeinhin überbewertet und er sähe in der Pille auch keine revolutionäre Sprengkraft. Innerhalb der Ehe angewendet, könne die Pille jedoch die Harmonie in der Familie fördern und die Frau von der Angst einer ungewollten Schwangerschaft befreien. Pincus verortete die Pille ebenfalls als wichtiges Mittel bei der ehelichen Familienplanung. Ihre Anwendung in ausserehelichen Beziehungen wurde daher gar nicht erst angesprochen.

In der Einleitung zum Interview mit Pincus wurde erneut auf die Rolle der Ärzte hingewiesen und betont, dass diese prüfen müssten, ob «[...] die Gründe, die gegen eine mögliche Schwangerschaft sprechen, wirklich stichhaltig sind» (ebd.). Die wiederholte Betonung verweist darauf, dass die Ärzte als Entscheidungsinstanz für Verhütung in der Annabelle nicht hinterfragt wurden. Zugleich wird im Beitrag eine problematische Überlagerung auf der Ebene der Sprechinstanz deutlich: Gregory Pincus wird als Koryphäe auf dem Gebiet der Fortpflanzung vorgestellt. Zugleich tritt er im Interview als Privatperson auf und äussert primär persönliche Einschätzungen zum Thema Verhütung. Für Frauen, die die Pille - aus welchen Gründen auch immer - nehmen wollten, bedeutete diese Machtposition der Ärzte eine paradoxe Situation: Das neue Verhütungsmittel versprach zwar mehr

50 Die letzten zwei Teile der Hormon-Serie wurden nicht in die Analyse miteinbezogen, da sie die Wechseljahre (Doppel-Nr. 414/415) sowie die längere Lebensdauer von Frauen (Nr. 417) thematisieren. 
Selbstbestimmung, diese Autonomie konnte jedoch nur mithilfe der Unterstützung eines Arztes erreicht werden. Was unter diesen «stichhaltigen Gründen» für eine Einnahme der Pille zu verstehen war, wird im Artikel nicht ausgeführt. Die Tatsache, dass die Pille bis zu diesem Zeitpunkt in der Annabelle nur im Kontext der Familienplanung besprochen wurde, erweckt den Eindruck, dass die Pille - aus Sicht der Zeitschrift - nur für verheiratete Frauen vorgesehen war, die ihre Mutterschaft im Rahmen der Ehe planen wollten. Somit unterstützt die Annabelle eine Abgabepraxis des Verhütungsmittels, die von einem grossen Teil der Schweizer Ärzteschaft gehandhabt und in deren Rahmen die Pille nur verheirateten Frauen verschrieben wurde. ${ }^{51}$ Diese Haltung wird gestärkt durch die Bebilderung des Artikels: Ein grosses Schwarz-Weiss-Foto dominierte die Seite 101, auf dem eine glückliche Mutter mit ihrem Neugeborenen zu sehen ist. Die Antibabypille soll von den Leserinnen der Annabelle nicht wörtlich verstanden werden als Mittel «gegen» Kinder, sondern lediglich garantieren, dass der Zeitpunkt der Mutterschaft geplant werden kann.

Weiter sprach Pincus das Bevölkerungswachstum - das in seinen Augen eine Gefahr darstellt - an: «Die Pille führt nicht zur Anarchie, sondern bedeutet für Völker, die einen gewissen Reifegrad erreicht haben, ein Mittel der Selbstdisziplin» (ebd., 103). Er führte aus, dass die Pille dabei helfen könne, den Lebensstandard in «Entwicklungsländern» zu heben. Solche Debatten mit Fokus auf die Verbindung von ökonomischen Überlegungen und der Pille waren in den 1960er Jahren sehr aktuell und daher erstaunt es nicht, dass diese Thematik auch in der Annabelle angesprochen wurde. Elisabeth Watkins weist in ihrem Werk zur Sozialgeschichte hormoneller Verhütungsmittel darauf hin, dass die Erfindung der Pille im Rahmen dieser Debatten als wissenschaftliche Lösung für das globale Bevölkerungswachstum angesehen wurde:

Demographers in the 1960s successfully focused the public's attention on the potential 'crisis' of overpopulation: in that climate, advocates of population control urged widespread use of birth control, including the pill, to slow population growth in the United States and abroad. 52

In den 1960er und 1970er Jahren wurde das orale Kontrazeptivum in Projekten der Entwicklungszusammenarbeit zur Geburtenkontrolle eingesetzt, um die Fruchtbarkeit armer Bevölkerungsschichten zu senken: «Gesellschaften

51 Diese Thematik besass auch später noch mediale Sprengkraft, wie folgendes Beispiel zeigt. Im Februar 1968 sorgte eine Aktion der Zürcher Studierenden Zeitung für grosses Aufsehen, da die Studierenden im zürcher student eine Liste mit Ärzten publizierten, die die Pille auch ledigen Frauen verschrieben (vgl. Joris/Witzig 20014, 297).

52 Watkins 1998, 2f. 
sollten sich 'nach Plan' reproduzieren - und das hiess, Kontrolle darüber auszuüben, wie andere Menschen ihre Körper kontrollierten..$^{53}$ Pincus bezeichnete die Pille als «Mittel zur Selbstdisziplin». Das Bevölkerungswachstum wurde von ihm also nicht nur als staatlich ökonomische Angelegenheit verstanden - und so an die Leserinnen der Annabelle vermittelt -, sondern auch als Teil der individuellen Verantwortung.

\section{Neue Kommunikationsstrategie: RatgeberInnen sprechen über Sexualität}

Ab dem Sommer 1967 wurden regelmässig Artikel über Empfängnisverhütung und Sexualität publiziert sowie vermehrt sexuelle Themen in Ratgeberrubriken besprochen. Dieser Wandel von der Nicht-Thematisierung von Sexualität zu einem offenen Sprechen über Sexualität - oft in einem Modus der Problemlösung - ist nicht nur in der Annabelle zu beobachten, sondern stellt eine gängige mediale Entwicklung zu dieser Zeit dar. ${ }^{54}$ Monica Suter weist darauf hin, dass Zeitschriften diese Ratgeberfunktion erst ab Mitte der 1960er Jahre wahrnahmen und Sexualität im öffentlichen Diskurs so präsenter wurde. ${ }^{55}$ Weiter führt sie aus, dass: «Jeder Winkel der Sexualität ausgeleuchtet und im Rahmen einer thematischen Auffächerung Themen salonfähig [wurden], die noch kurz zuvor tabuisiert gewesen waren.» ${ }^{56}$

Aus heutiger Perspektive wird deutlich, dass die 1966 veröffentlichte Studie «Human Sexual Response» vom amerikanischen Forschungsteam William H. Masters und Virginia E. Johnson den Umgang mit Sexualität in Ratgebern massgeblich beeinflusste. Diese Untersuchungen zum menschlichen Sexualverhalten, in denen die physiologische Reaktion des männlichen sowie weiblichen Körpers während des Orgasmus getestet wurden, widerlegten viele herrschende Vorstellungen über Sexualität und waren prägend für weitere Publikationen in diesem Bereich. Masters und Johnson konzentrierten sich auf Psychologie sowie Physiologie des menschlichen Sexualverhaltens und vertraten die Haltung, dass «sexuelle Probleme» behandelt werden konnten. Sexualität wurde in den Medien nun vornehmlich in einem Modus der Problemlösung betrachtet und eine Abgrenzung zur psychoanalytischen Sexualitätskonzeption zeichnete sich ab. ${ }^{57}$

53 Niethammer/Satjukow 2016, 12.

54 Vgl. Bänziger/Beljan/Eder 2015, 12.

55 Vgl. dazu bspw. auch Lutz Sauerteigs Forschung zur deutschen Jugendzeitschrift Bravo: Sauerteig 2010.

56 Suter 1996, 146

57 Vgl. Bänziger/Beljan/Eder et al., 12. 
In der Annabelle wurden sexuelles Verhalten oder Praktiken nicht im Detail thematisiert, sondern vielmehr grundlegende Einstellungen zum Sexualverhalten und einem moralischen Lebenswandel besprochen, sowie Empfehlungen dazu abgegeben. Im Mai 1967 positionierte sich die Annabelle erstmals deutlich zur ausserehelichen Verwendung der Pille und kommunizierte nicht mehr nur über Experten aus der Medizin und Psychologie, wie in den ersten Artikeln. Nun waren die Chefredaktorin Eva Maria Borer sowie Dr. Fritz Tanner als «populärmediale Ratgeber ${ }^{58}$ wortführend. Tanner publizierte neben seiner Tätigkeit als Psychotherapeut zahlreiche Werke zu Ehe, Beziehung, Sexualität sowie Erziehung und trat als Sexualberater im Fernsehen auf. Als Mitglied des Nationalrats (1967-1974) setzte er sich unter anderem für das Frauenstimmrecht und die Einführung eines Sexualkundeunterrichts ein. Trotz seiner Fachkenntnisse gab sich Tanner in seinen Kolumnen nicht wissenschaftlich distanziert, sondern war um Nahbarkeit bemüht.

Im Rahmen einer Kolumne bezog Tanner Stellung zur Verwendung der Pille: «Allen, die in ausserehelichen Sexualbeziehungen stehen, ist sie [die Pille] selbstverständlich nicht weniger willkommen. Sie sehen in ihr vielmehr die langersehnte, 'endlich' eingetroffene Möglichkeit einer risikolosen, freien Liebesbegegnung» (Nr. 427, Mai 67, 12). Er verteidigte seinen Standpunkt, indem er darauf hinwies, dass es besser sei, ledigen Frauen die Pille zu verschreiben, als sie einem potentiellen Schwangerschaftsabbruch auszusetzen. Die Einstellung des Therapeuten zur Pille und «freien Liebesbegegnungen» teilten jedoch nicht alle Leserinnen, wie sich in den Reaktionen auf den Artikel «Ferienflirt für Anfängerinnen» (Nr. 430, Juli 67) zeigt. Grund für die Entrüstung der Leserschaft war der Schlusssatz des Artikels, in dem der Tipp gegeben wurde: «Wenn ihr's schon nicht lassen könnt - vergesst wenigstens die Pille nicht» (ebd., 55). In mehreren Leserbriefen wurde die Beschwerde angebracht, dass diese Art der Berichterstattung zu weit gehe. Chefredaktor Hans Gmür entschuldigte sich darauf in einer Stellungnahme bei seiner Leserschaft für den fehlgeleiteten Ferientipp (vgl. Nr. 434, Aug 67, 119). Diese Reaktion zeigt, dass die neue, offenere Kommunikationsstrategie in der Annabelle für gewisse Unsicherheiten sorgte und eine klare Haltung zu solch polarisierenden Inhalten noch gefunden werden musste.

In der Antwort von Eva Maria Borer auf einen Leserbrief in der Septemberausgabe von 1967 zeigt sich, wie sich das Sprechen über Sexualität in der Annabelle in dieser kurzen Zeitspanne gewandelt hat. Borer nahm eine moralisierend-psychologisierende Sprecherrolle ein und trat als entschiedene

58 Bänziger 2010, 54. 
Beraterin auf. In einem Leserbrief beschwerte sich eine junge Frau über das veränderte Sexualverhalten ihrer Altersgenossinnen und kritisierte, dass diese von Männern immer gleich «einen Sexualverkehr verlangen» (Nr. 435, Sept 67, 12). Borer gab zur Antwort, dass «Die Mädchen, von denen Sie sprechen, [...] unausgeglichen und unzufrieden [sind]» (ebd.) und nicht gelernt hätten, mit ihren Freiheiten umzugehen. Damit formuliert sie eine negative Wertung dieser «schnellen Sexualität», nur in Verbindung mit Liebe könne «echte» Sexualität («mit Leib und Seele») erfahren werden. Weiter erwähnte sie, dass einige dieser Mädchen unter Nymphomanie leiden würden und pathologisierte somit ihr Sexualverhalten. Sie betonte jedoch auch, dass diese Mädchen eine Ausnahme seien. Borer als lauteste «Stimme» der Annabelle berichtete ausführlich über Sexualität und beriet ihre Leserinnen offen. Bei all dieser Offenheit wurde jedoch immer wieder betont, dass die «richtige» Sexualität in der Ehe oder einer längeren, stabilen Beziehung gelebt werden solle und eine zu freie, weibliche Sexualität verfehlt sei. Diese Vermittlung bürgerlicher Moralvorstellungen - die auch von einem Teil der Leserschaft eingefordert wurde -, steht in einem Spannungsverhältnis zur Publikation von Artikeln in der Annabelle, in denen aussereheliche Sexualität nicht nur thematisiert, sondern ausdrücklich befürwortet wurde.

In der Doppelausgabe vom November 1967 nahm der Eheberater Fritz Tanner eine Einschätzung zur medialen Berichterstattung über Sexualität vor und verriet dabei einiges über seine persönliche Einschätzung sowie das Selbstbild der Annabelle. Tanner sah die «Aufgabe des sexualpsychologischen Journalismus» darin, Aufklärung zu betreiben ohne «den menschlichen Sex zur Pressesensation zu machen» (Nr. 440/441, Nov 67, 21). Er betrachtete das Sprechen über Sexualität als Voraussetzung für eine funktionierende Beziehung und widersprach dem Vorwurf, Berichte über Sexualität aus ökonomischen Beweggründen zu publizieren. Somit setzte er sich in der Annabelle für einen ungezwungenen Umgang mit Sexualität und der Aufklärung darüber ein.

Im Editorial der 442. Ausgabe verteidigte auch Chefredaktor Hans Gmür die breite Berichterstattung über Sexualität und sah es als Pflicht, dass in der Annabelle «alle Probleme unserer Zeit» - und dazu gehörten auch Probleme im sexuellen Bereich - besprochen werden konnten. Diese Wortmeldungen können im Kontext der ab Mitte der 1960er Jahre diskutierten «Sexwelle» verortet werden. Zu dieser Zeit setzte eine Reihe von Zeitschriften und Zeitungen erfolgreich auf das Motto «sex sells» und berichtete regelmässig über sexuelle Themen, oft kombiniert mit erotischen Bildern. ${ }^{59}$

59 Eder 2015, 38f. 
Im neuen Jahr änderte sich der Ton in der Annabelle bezogen auf Sexualität und Verhütung nicht stark und so standen Fragen, wie beispielsweise nach dem «normalen» sexuellen Verhalten oder der moralischen Aspekte davon weiterhin im Vordergrund. Nachdem das Thema der hormonellen Verhütung 1967 nicht mehr so prominent besprochen wurde, war ihr im März 1968 ein dreiseitiger Artikel gewidmet. Darin berichtete die amerikanische Schriftstellerin Pearl S. Buck über den Einfluss der Pille auf die Moralanschauungen. Sie stellte verschiedene Standpunkte zur Thematik vor und ergänzte diese jeweils mit ihrer persönlichen Meinung. Sie beschrieb, dass sie voreheliche sexuelle Beziehungen aufgrund einer möglichen Schwangerschaft immer kritisch eingeschätzt habe, dieser Einwand durch die Pille jedoch aufgehoben sei. Wie Borer sei sie der Meinung, dass Sex ohne Liebe schädigend sein könne und definierte eine «neue Moral»: «Die Wirkung des Missbrauchs [Reduzierung der Sexualität auf physischen Genuss] bringt eine Schädigung der ganzen Persönlichkeit mit sich und kann schliesslich sogar die Ehe ihres tieferen Sinns und ihrer vollen Bedeutung berauben» (Nr. 448, März 68, 9). Sie mass der Pille - wie auch im einleitenden Zitat dieser Arbeit zum Ausdruck kommt - enorme Bedeutung zu im Bezug auf Veränderungen der Sexualmoral und bemerkte, dass die Auswirkungen der Pille auf die Gesellschaft zu einer «sozialen Bombe» geworden seien (ebd., 8).

In der Vorbemerkung zum Beitrag betonte Borer, wie wichtig sie den Ansatz von Pearl S. Buck fände, sich mit dem Einfluss der Pille auf bestehende Moralvorstellungen auseinanderzusetzen - und nicht etwa lediglich gesundheitliche Risiken oder ihre Anwendungsform zu diskutieren. Sie verweist zudem darauf, dass die möglichen Auswirkungen des Verhütungsmittels auf «Begriffe von Gut und Böse» (ebd.) im Zentrum der Berichterstattung stehen sollten. Borer impliziert, dass die Pilleneinnahme ein moralisches Problem darstellen kann. Dabei macht sie deutlich, dass «ihrer» Zeitschrift und insbesondere ihr selbst als Ratgeberin bei der Lösung dieses Problems eine wichtige Rolle zukommt.

\section{Fazit}

In der Untersuchungsperiode hat sich das Sprechen über die Antibabypille sowie über Sexualität in der Annabelle grundlegend gewandelt: Von einer anfänglichen Nicht-Thematisierung bis zu einer regen Berichterstattung im Modus der Problemlösung. Anfang 1966 kann in der Annabelle eine sehr zurückhaltende Berichterstattung über Sexualität beobachtet werden, die sich jedoch 
ab Mitte 1967 rasch und dynamisch entwickelte. So fand die erste Thematisierung von Sexualität in einem Duktus der Abgrenzung statt, in dem über amerikanische und daher «fremde» Sexualität berichtet wurde. Dass die Annabelle dabei Experten aus der Wissenschaft sprechen lässt, ist charakteristisch für das Sprechen über Sexualität in den 1960er Jahren. Auch in der Artikelserie über die Wirkungsweise von Hormonen zeigt sich sprachlich und inhaltlich eine stark wissenschaftliche Prägung. So wurde die Pille als hoch komplexes Medikament beschrieben. Frauen als Anwenderinnen kommen dabei nicht zu Wort und nach Erfahrungsberichten über das hormonelle Kontrazeptivum sucht man Mitte der 1960er Jahre in der Annabelle vergeblich.

In dieser Phase wurde die Pille ausschliesslich im Kontext der Familienplanung vorgestellt und wiederholt betont, wie wichtig eine ärztliche Einschätzung in diesem Bereich sei. Die Berichterstattung über Verhütung im Rahmen von Familienplanung kann als Anpassungsstrategie gedeutet werden, mit der die Annabelle versuchte, über ein neues Thema wie die Antibabypille zu sprechen, ohne den Rahmen eines bürgerlichen Moralkonsensus verlassen zu müssen. Die Frauenzeitschrift beschreitet so einen Mittelweg zwischen bewährter und moderner Ausrichtung - sehr wahrscheinlich auch um eine breite Leserschaft anzusprechen und so auf die Konkurrenzsituation auf dem Zeitschriftenmarkt zu reagieren. Die Annabelle war zu Beginn der 1960er Jahre praktisch konkurrenzlos, gegen Ende des Jahrzehnts erfreuten sich jedoch immer mehr ausländische Frauenzeitschriften der Beliebtheit der Schweizer Leserschaft. Um einen Wachstumseinbruch zu verhindern, besetzte sie vermehrt gesellschaftspolitisch aktuelle Themen, hielt aber zugleich an einem konservativen Wertekanon fest, um die Stammleserschaft nicht zu verlieren. $\mathrm{Zu}$ dieser Zeit war das Thema Sexualität in der medialen Öffentlichkeit sehr präsent und die Berichterstattung in der Annabelle im Vergleich zu anderen Medienerzeugnissen eher zurückhaltend. Ende der 1960er Jahre kann im deutschsprachigen Raum einerseits eine gelockerte Berichterstattung über Sexualität - eine «Sexwelle» - in Illustrierten festgestellt werden, zugleich gibt es auch Zeitschriften, die dezidiert Abstand nahmen von einer solchen Thematisierung. Eine Strategie, die - wie das Beispiel der Bunte in Deutschland zeigt - durchaus erfolgreich sein konnte. ${ }^{60}$

Nach dieser Phase wissenschaftlich geprägter Artikel kamen jedoch bald andere Stimmen - mit Eva Maria Borer erstmals eine weibliche - zu Wort. Ab Mitte 1967 kann eine neue Kommunikationsstrategie festgemacht werden, in der sich die Redaktion der Annabelle in sexuellen Belangen direkt an 
ihre Leserschaft wendete und im Modus der Problemlösung über Sexualität berichtete. Das Sprechen über Sexualität als Aufklärung oder in Form von Ratschlägen gehörte ab Ende der 1960er Jahre zum Selbstbild der Annabelle, womit sie einem zeitgenössischen medialen Trend folgte. In diesem Zusammenhang wurde die Pille nicht mehr nur im Kontext der Familienplanung thematisiert, sondern auch in Verbindung mit ausserehelicher Sexualität. ${ }^{61}$ Trotz dieser thematischen Öffnung, die der problemlösende Duktus mit sich brachte, positionierte sich die Annabelle durch Anleitungen zum «richtigen» (in Verbindung mit Liebe, in einer festen Beziehung) und «falschen» (wechselnde Partner, Fokus auf Lustempfinden) Verhalten sehr deutlich und vermittelte ihren Leserinnen weiterhin das Ideal einer innerhalb der Ehe gelebten Sexualität. Trotz des Wertewandels der immer wieder in Zusammenhang mit der Pille heraufbeschworen wird, ob im Sinne der Familienplanung oder Selbstbestimmung über den weiblichen Körper, wird die Ehe noch immer als «höchste Beziehungsform für sexuelle Erfahrungen ${ }^{62}$ angesehen. Andere Beziehungsformen hingegen wurden im Vergleich dazu als defizitär bewertet. Die Quellenanalyse hat gezeigt, dass die Annabelle auch in der Phase des gelockerten Sprechens über Verhütung und Sexualität weiterhin am Ehemodell als Ort der gelebten Sexualität festhielt.

\section{Bibliographie}

Anderegg, Susanne, Das Frauenbild der Zeitschriften «Annabelle» und «Meyers Modeblatt»: Entwicklung seit dem zweiten Weltkrieg (Zürich 1987) (unveröff. Lizentiatsarbeit der Universität Zürich)

Bänziger, Peter-Paul, Sex als Problem. Körper und Intimbeziehungen in Briefen an die «Liebe Marta» (Frankfurt am Main 2010) (Campus Forschung 948)

Bänziger, Peter-Paul/Duttweiler, Stefanie/Sarasin, Philipp et al. (Hrsg.), Fragen Sie Dr. Sex! Ratgeberkommunikation und die mediale Konstruktion des Sexuellen (Berlin 2010) (edition suhrkamp 2595)

Bänziger, Peter-Paul/Magdalena Beljan/Franz X. Eder et al. (Hrsg.), Sexuelle Revolution? Zur Geschichte der Sexualität im deutschsprachigen Raum seit den 1960er Jahren (Bielefeld 2015) (Kulturgeschichte der Moderne 9)

Christen, Mariana/Johann Gisler/Martin Heller (Hrsg.), Ganz Annabelle. Eine Zeitschrift als Freundin (Zürich 1992)

Duden, Barbara, «Von 'der' Pille und unserem 'Zustand'», in: Gisela Staupe/Lisa Vieth (Hrsg.), Die Pille. Von der Lust und von der Liebe (Berlin 1996) (Publikation des Deutschen Hygiene Museums, Dresden) 67-81

Eder, Franz X, «Die lange Geschichte der 'Sexuellen Revolution' in Westdeutschland (1950er bis 1980er Jahre)», in: Peter-Paul Bänziger/Magdalena Beljan/Franz X. Eder et al. (Hrsg.), Sexuelle Revolution? Zur Geschichte der Sexualität im

61 Vgl. Bänziger/Duttweiler/Sarasin 2010, 10f.

62 Joris/Witzig 20014, 294. 
deutschsprachigen Raum seit den 1960er Jahren (Bielefeld 2015) (Kulturgeschichte der Moderne 9) 25-59

Eder, Franz X., «Das Sexuelle beschreiben, zeigen und aufführen. Mediale Strategien im deutschsprachigen Sexualdiskurs von 1945 bis Anfang der siebziger Jahre», in: Peter-Paul Bänziger/Stefanie Duttweiler/Philipp Sarasin et al. (Hrsg.), Fragen Sie Dr. Sex! Ratgeberkommunikation und die mediale Konstruktion des Sexuellen (Berlin 2010) 94-123 (edition suhrkamp 2595)

Gisler, Johanna/Mariana Christen, «Die 'Schule der Frauen'. Zur Modernisierung des Frauenleitbildes in der Nachkriegszeit», in: Andreas Ernst/Thomas Gerlach/ Patrick Halbeisen et al. (Hrsg.), Kontinuität und Krise. Sozialer Wandel als Lernprozess. Beiträge zur Wirtschaftsund Sozialgeschichte der Schweiz (Zürich 1994) 181-207 (Festschrift für Hansjörg Siegenthaler)

Hediger, Melanie, Das Bild der Schweizer Frau in Schweizer Zeitschriften. Studien zu «Annabelle», «Schweizer Illustrierte» und «Sonntag» von 1966 bis 1976 (Fribourg 2004)

Joris, Elisabeth/Heidi Witzig (Hrsg.), Frauengeschichte(n). Dokumente aus zwei Jahrhunderten zur Situation der Frauen in der Schweiz (Zürich ${ }^{42001)}$

Junod, Suzanne White/Lara Marks, Women's Trials. The Approval of the First Oral Contraceptive in the United States and Great Britain (Oxford 2002) 117-160 (Journal of the History of Medicine and Allied Sciences 57, 2/2002)

Lengwiler, Martin/Madarász, Jeanette, «Präventionsgeschichte als Kulturgeschichte der Gesundheitspolitik», in: Martin Lengwiler/Jeanette Madarász (Hrsg.), Das präventive Selbst. Eine Kulturgeschichte moderner Gesundheitspolitik ( Bielefeld 2010) 11-28 (Verkörperungen/Matterealities 9)

Marks, Lara, Sexual Chemistry. A History of the Contraceptive Pill (New Haven 2001)

Minetti, Katja, Intimität in Kontaktanzeigen: linguistische diachrone Untersuchung über die Herstellung und Funktion von Intimität in Kontaktanzeigen aus der Annabelle-Zeitschrift von 1955 bis 2005 (Zürich 2016) (unveröff. Lizentiatsarbeit der Universität Zürich)

Niethammer, Lutz/Silke Satjukow (Hrsg.), «Wenn die Chemie stimmt...»: Geschlechter-beziehungen und Geburtenkontrolle im Zeitalter der «Pille» (Göttingen 2016)

Peter-Kubli, Susanne, Fritz Tanner, in: Historisches Lexikon der Schweiz (HLS) online: http://www.hls-dhs-dss.ch/textes/d/D6704.php [Stand 31.08.2018]

Sauerteig, Lutz, «'Wie soll ich es nur anstellen, ohne etwas falsch zu machen?' Der Rat der Bravo in Sachen Sex in den sechziger und siebziger Jahren, in: Peter-Paul Bänziger/Stefanie Duttweiler/Philipp Sarasin et al. (Hrsg.), Fragen Sie Dr. Sex! Ratgeberkommunikation und die mediale Konstruktion des Sexuellen (Berlin 2010) 94-123 (edition suhrkamp 2595)

Silies, Eva-Maria, «Befreiung oder Bürde. Erfahrungen von Frauen mit der Pille in der Bundesrepublik der langen 1960er Jahre», in: Lutz Niethammer/Silke Satjukow (Hrsg.), «Wenn die Chemie stimmt...»: Geschlechterbeziehungen und Geburtenkontrolle im Zeitalter der 'Pille' (Göttingen 2016) 193-210

Staupe, Gisela/Lisa Vieth, Die Pille. Von der Lust und von der Liebe (Berlin 1996) (Publikation des Deutschen Hygiene Museums, Dresden)

Steinbacher, Sybille, Wie der Sex nach Deutschland kam. Der Kampf um Sittlichkeit und Anstand in der frühen Bundesrepublik (München 2011) 
Suter, Monica, Pille, Familienplanung und die Medikalisierung der Empfängnis-verhütung. Der medizinische Diskurs über Empfängnisverhütung in der Schweiz (1960-1975) ( Zürich 1996) (unveröff. Lizentiatsarbeit der Universität Zürich)

Tanner, Jakob, Geschichte der Schweiz im 20. Jahrhundert (München 2015)

Wajcman, Judy, Technik und Geschlecht. Die feministische Technikdebatte (Frankfurt am Main/New York 1994)

Watkins, Elisabeth, On the Pill: A Social History of Oral Contraceptvices, 19501970 (Baltimore 1998) 